\title{
Actitudes, dudas y conocimientos de los colectivos implicados en la atención del paciente con Alzheimer: resultados de la encuesta del proyecto kNOW Alzheimer
}

\author{
Pablo Martínez-Lage Álvarez \\ en representación del "Grupo de estudio del proyecto kNOW Alzheimer"*
}

\section{* Integrantes del Grupo de estudio del proyecto kNOW Alzheimer:}

Pablo Martínez-Lage Álvarez Departamento de Neurologia, Centro de Investigación y Terapias Avanzadas de la Fundación CITA-Alzheimer, San Sebastián. Enrique Arrieta-Antón Centro de Salud Segovia Rural, Segovia. Coordinador del Grupo de Trabajo de Neurologia de la Sociedad Española de Médicos de Atención Primaria (SEMERGEN). Juan José Gomáriz-García Centro de Salud Lorca Sur, Lorca, Murcia, España. Miembro del Grupo de Trabajo de Neurología de la SEMERGEN. Enrique Arriola-Manchola Unidad de Memoria y Alzheimer, Fundación Matia, San Sebastián. Coordinador del Grupo de Demencias de la Sociedad Española de Geriatría y Gerontología (SEGG). José Manuel Marin-Carmona Programa Municipal de Memoria y Demencias, Avuntamiento de Málaga. Presidente de la Sociedad Andaluza de Geriatría y Gerontología. Rosa Brescané-Bellver Confederación Española de Asociaciones de Familiares de Personas con Alzheimer y otras Demencias (CEAFA). Gemma Tomé-Carruesco Asistente Social, Hospital Mutua de Terrassa, Barcelona. Guillermo García-Ribas Servicio de Neurologia, Hospital Universitario Ramón y Cajal, Madrid. Coordinador del Grupo de Estudio de Demencias y Neurologia de la Conducta de la SEN. María Sagrario Manzano-Palomo Servicio de Neurología, Hospital Infanta Cristina, Parla, Madrid, España. Secretaria del Grupo de Demencias de la SEN. Rosa María Rodríguez-Fernández Servicio de Neurología, Complexo Hospitalario Universitario de Ourense. Ana María Molinero Crespo Vicepresidenta de la Sociedad Española de Farmacia Familiar y Comunitaria (SEFAC). Laura Tuneu-Valls. Servicio de Farmacia Hospitalaria, Hospital de la Santa Creu i Sant Pau, Barcelona.

\section{PALABRAS CLAVE}

Enfermedad de Alzheimer, profesionales sanitarios, cuidadores, conocimiento, actitudes, necesidades formativas

\section{ABREVIATURAS}

CEAFA: Confederación Española de Familiares de Personas con Alzheimer y otras Demencias

EA: enfermedad/enfermo de Alzheimer IMPACT: Important Perspectives on Alzheimer's Care and Treatment MAP: médico de atención primaria

MMSE: mini mental state examination NIA-AA: National Institute on Aging and the Alzheimer's Association SEFAC: Sociedad Española de Farmacia Familiar y Comunitaria SEGG: Sociedad Española de Geriatría y Gerontología SEMERGEN: Sociedad Española de Médicos de Atención

Primaria

SEN: Sociedad Española de Neurología

\section{RESUMEN}

Introducción: La enfermedad de Alzheimer (EA) involucra a colectivos diversos de profesionales y cuidadores. Las actitudes y necesidades de formación en diagnóstico temprano, tratamiento, síntomas conductuales, recursos sociosanitarios y aspectos legales o éticos son desconocidas.

Métodos: Estudio observacional transversal tipo encuesta dentro del proyecto kNOW Alzheimer, iniciativa de la SEN, SEGG, SEMERGEN, SEFAC y CEAFA mediante cuestionarios específicos. Cada sociedad invitó a todos sus miembros a cumplimentarlos a través de www.knowalzheimer.com.

Resultados: Participaron 114 neurólogos, 113 geriatras, 275 médicos de atención primaria, 328 farmacéuticos y 858 cuidadores. Los retrasos en el diagnóstico se originan en pacientes y cuidadores, el sistema y en los profesionales, que carecen de medios o malinterpretan sintomas tempranos. Persiste el uso del término "demencia senil". Profesionales y cuidadores perciben buena eficacia del tratamiento. Se apuesta por el diagnóstico y tratamiento en fase pre-demencia. Hay déficit de formación en el manejo de la conducta. Los profesionales afirman que informan pero los cuidadores se sienten desinformados. Los cuidadores desean conocer cuanto antes si ellos o un familiar padecen Alzheimer. Los sintomas conductuales y la necesidad de restringir actividades son fuentes de sobrecarga. Los farmacéuticos pueden contribuir a la detección y manejo, pero precisan de formación amplia. Hay necesidad de formación en aspectos legales, bioéticos, recursos y acceso a la investigación.

Conclusiones: Este proyecto ha permitido obtener información sobre actitudes y dudas de los colectivos involucrados en la atención a la EA, necesidades de formación y puntos de mejora.

Financiación: La estructura informática, el mantenimiento de la base de datos de kNOW Alzheimer y el soporte logistico del proyecto fueron financiados por STADA Arzneimittel GmbH. Ninguna de las personas de STADA Arzneimittel $\mathrm{GmbH}$ ha participado, opinado, influido ni modificado de manera alguna el diseño del estudio, interpretación de los resultados ni la elaboración del presente manuscrito.

Conflicto de intereses: Los autores declaran no existir conflicto de intereses en relación con el contenido del articulo.

Cite este artículo como: Martinez-Lage P, et al. Actitudes, dudas y conocimientos de los colectivos implicados en la atención del paciente con Alzheimer: resultados de la encuesta del proyecto kNOW Alzheimer. Farmacéuticos Comunitarios. 2016 Mar 01; 8(1):13-23. doi:10.5672/FC.2173-9218.(2016Nol8).001.03

Correspondencia: Pablo Martinez-Lage Álvarez (pmlage@cita-alzheimer.org).

ISSN 1885-8619 @ SEFAC (Sociedad Española de Farmacia Familiar y Comunitaria). Todos los derechos reservados. 
Attitudes, uncertainties and knowledge among the different parties involved in alzheimer's patients care: findings from the kNOW Alzheimer project survey study

\section{KEYWORDS}

\section{Alzheimer}

disease, sanitary

professionals,

caregivers,

knowledge,

attitudes, training

needs

\section{ABSTRACT}

Background: Alzheimer's disease (AD) involves different groups of professionals and caregivers. Their attitudes and needs of training in early diagnosis, treatment, behavioral symptoms, resources, social and legal or ethical aspects are unknown.

Methods: Survey study within the project "kNOW Alzheimer”, a SEN, SEGG, SEMERGEN SEFAC and CEAFA initiative, through specific questionnaires. Each Society invited all its members to complete them through www.knowalzheimer.com.

Results: Participants were 114 neurologists, geriatricians 113, 275 primary care physicians, 328 pharmacists and 858 caregivers. Delays in diagnosis arise in patients and caregivers, the system and the professionals, who lack the means or misinterpreted early symptoms. The term "senile dementia" is still in use. Professionals and caregivers perceived good efficacy of treatment. Professionals advocate for diagnosis and treatment in the pre-dementia stage. There is a need for training in behavior management. Practitioners claim they inform but caregivers feel uninformed. Caregivers want to know as soon as possible if they or a family member suffers from $\mathrm{AD}$. Behavioral symptoms and the need to restrict activities are sources of overload. Pharmacists can contribute to detection and management, but require extensive training. There is a need for training on legal aspects, bioethics, resources and access to research.

Conclusions: This project has yielded information on attitudes and doubts of the collectives involved in $\mathrm{AD}$ care, their training needs and points of improvement.

\section{Introducción}

La demencia en general, y la enfermedad de Alzheimer (EA) en particular, es una patología extraordinariamente prevalente y peculiar que exige para su adecuada atención y cuidado la participación de diversos grupos de profesionales de las redes sociosanitarias, así como de los familiares, cuidadores y de los propios pacientes. La EA afecta en España a una de cada diez personas mayores de 65 años (1) y repercute en la vida diaria de 3,5 millones de individuos, entre familiares y cuidadores. Dado el envejecimiento progresivo de la población se prevé que en el año 2020 se duplicará el número de enfermos y que la cifra de afectados podría llegar a 1.500 .000 casos en 2050 (2).

La actitud de los profesionales médicos frente al diagnóstico temprano y su percepción sobre la eficacia de los tratamientos farmacológicos y no farmacológicos disponibles pueden influir significativamente en el manejo adecuado de los pacientes (3). La dificultad que entraña la identificación correcta de los primeros sintomas por parte de los propios pacientes y allegados, las circunstancias reales de trabajo de los profesionales de atención primaria, las vicisitudes de coordinación entre atención primaria y especializada, la disponibilidad de los medios diagnósticos, la utilización o no de biomarcadores y otras técnicas diagnósticas, así como numerosos factores pueden explicar la variabilidad en el retraso en el diagnóstico de la enfermedad e instauración de un tratamiento adecuado (4-12).

Además, y entre otros, se añaden dilemas relacionados con la comunicación del diagnóstico, asesoramiento ético y legal, papel de los profesionales de la medicina en el seguimiento de pacientes y familiares durante el curso de la enfermedad, supresión del tratamiento o toma de decisiones al final de la vida (13-15). Por otra parte, tampoco se ha analizado el posible papel de los profesionales de las farmacias comunitarias, a los que los usuarios tienen un acceso más directo, y su posible contribución a la detección temprana y tratamiento adecuado de la enfermedad. A pesar de las directrices formuladas por la Comisión Europea para impulsar la aprobación de programas específicos de atención e investigación en los estados miembros de la Unión $(16,17)$, España sigue siendo un país europeo carente de un plan nacional para la atención de los pacientes con demencia y EA.

Con el fin de obtener datos actualizados que permitan una aproximación a la realidad de la atención a la EA en nuestro país, se diseñó un estudio transversal de tipo encuesta. El objetivo fue conocer las actitudes de los distintos colectivos involucrados en el diagnóstico y manejo de los pacientes con EA, detectar sus necesidades de formación y analizar las circunstancias susceptibles de mejora que pudieran redundar en un mayor beneficio en la atención a los pacientes y familiares o cuidadores.

\section{Material y métodos}

Estudio observacional transversal realizado entre el 21 de julio de 2012 al 30 de abril de 2013.

Mediante una encuesta dirigida a neurólogos, geriatras, médicos de atención primaria, farmacéuticos $\mathrm{y}$ familiares/cuidadores implicados en atender y tratar a pacientes con EA. El estudio se encuadra en el proyecto denominado kNOW Alzheimer. Dicho proyecto surge como iniciativa conjunta de la Sociedad Española de Neurología (SEN), Sociedad Española de Geriatría y Gerontología (SEGG), Sociedad Española de Médicos de Atención Primaria (SEMERGEN), Sociedad Española de Farmacia Familiar y Comunitaria (SEFAC) y Confederación Española de Familiares de Personas con Alzheimer y otras Demencias (CEAFA), contando con el aval de todas ellas y el soporte financiero y logístico de Laboratorio STADA.

El proyecto kNOW Alzheimer consta de dos fases, la primera dirigida a detectar aspectos del manejo general de la enfermedad sobre los que existen más dudas o controversias y, la segunda, a elaborar un manual detallado y específico para cada colectivo, enfocado a las lagunas de formación y necesidades de información detectadas en la primera fase, que servirá de elemento de referencia y consulta para los profesionales sanitarios y familiares/cuidadores en 
todos los aspectos relacionados con la EA.

Para el desarrollo de la primera fase se constituyó un comité científico del proyecto, en el seno del cual se establecieron cinco grupos de trabajo formados por profesionales de cada área designados por cada una de las sociedades participantes en el estudio. El propósito de cada grupo de trabajo era desarrollar un cuestionario orientado específicamente a su colectivo dirigido a valorar actitudes y necesidades de formación en aspectos de la atención a la EA relacionados con el diagnóstico, abordaje terapéutico, comunicación del diagnóstico, comorbilidades, asesoramiento legal o ético o acceso a la investigación. Cada cuestionario fue revisado por el coordinador del proyecto y aprobado posteriormente por el comité científico y las juntas directivas de cada sociedad. Una vez consensuado cada cuestionario se puso a disposición de todos los profesionales y cuidadores de cada colectivo por diversos medios, incluyendo el formato digital mediante la web del proyecto en www.knowalzheimer.com y también mediante el reparto directo de cuestionarios en formato impreso por parte de la red comercial de STADA.

Asimismo, se contó con la participación de las cinco sociedades implicadas para dar la máxima difusión al proyecto, de modo que cada una de ellas envió información solicitando la participación en el proyecto a todos aquellos miembros para los cuales dichas sociedades contaban con el permiso de uso de sus respectivos correos electrónicos. La participación estaba abierta a cualquier profesional y familiar/cuidador de los cinco colectivos indicados, sin más limitaciones, de forma libre y no incentivada. Además, se habilitó una página web en http://www.knowalzheimer.com para proporcionar información y aumentar la difusión del proyecto.

Las encuestas para cada colectivo constaban de una primera parte explicando los propósitos y características generales del proyecto $k N O W$ Alzheimer, seguida de un número variable de preguntas (entre 41 para farmacéuticos y 76 para cuidadores/ familiares). En todos los cuestionarios se incluía un primer apartado de datos sociodemográficos y una sección de preguntas específicas en las cuales había que escoger una opción entre cinco o tres posibles respuestas o indicar si se estaba totalmente en desacuerdo, parcialmente en desacuerdo, no sabe/no contesta (NS/NC), parcialmente de acuerdo o totalmente de acuerdo con un determinado enunciado. En la última parte, cada participante podía expresar su opinión sobre aspectos no recogidos en la encuesta o sugerencias para disponer de un mejor material de formación y consulta.

Los detalles de los cuestionarios para cada colectivo se pueden consultar en http://knowalzheimer.com/ profesionales/informes-encuesta/. Los cuestionarios se cumplimentaban utilizando la plataforma digital del proyecto y de forma anónima.

Se presentan los datos correspondientes a la estadística descriptiva.

\section{Resultados}

El número total de miembros de cada sociedad que se registraron en la plataforma del proyecto kNOW Alzheimer fue de 149 correspondientes a la SEN, 205 a la SEGG, 384 a la SEMERGEN, 460 a la SEFAC y 1.231 a la CEAFA. Para cada colectivo el número final de encuestas cumplimentadas y válidas fue de 114 (76,5\%) para el caso los neurólogos, 135 (55,1\%) para los geriatras, $275(71,5 \%)$ para los médicos de atención primaria, 328 $(71,3 \%)$ para los farmacéuticos y 825 $(69,7 \%)$ para los familiares/cuidadores. Los resultados principales para cada colectivo se detallan a continuación.

\section{Neurólogos}

Los datos demográficos y el ámbito de actividad de los especialistas en Neurología que respondieron a la encuesta se detallan en la tabla 1 . Un 21\% de los neurólogos ejercía su actividad en una unidad especializada y un 16\% lo hacía en consultas monográficas de demencias. Un 39\% de especialistas manifestaba disponer de solo 30 minutos para una primera

Tabla 1 Datos demográficos de los profesionales que han respondido a la encuesta

\begin{tabular}{|c|c|c|c|c|c|}
\hline & Neurólogos & Geriatras & Atención Primaria & Farmacéuticos & Cuidadores \\
\hline N & 114 & 113 & 275 & 328 & 858 \\
\hline Sexo (\% mujeres) & 48 & 57 & 59 & 71 & 77 \\
\hline \multicolumn{6}{|l|}{ Edad (\%) } \\
\hline$<30$ & 11 & 12 & 18 & 16 & 6 \\
\hline $30-40$ & 44 & 31 & 20 & 30 & 14 \\
\hline $40-50$ & 25 & 24 & 20 & 30 & 30 \\
\hline $50-60$ & 18 & 31 & 40 & 21 & 27 \\
\hline$>60$ & 3 & 3 & 2 & 3 & 24 \\
\hline Ámbito (\% rural) & 7 & 15 & 26 & 30 & \\
\hline \multicolumn{6}{|l|}{ Actividad (\%) } \\
\hline Hospital & 70 & 53 & & & \\
\hline Ambulatorio & 48 & 21 & & & \\
\hline Consulta de demencias & 16 & 16 & & & \\
\hline Unidad de demencias & 21 & 41 & & & \\
\hline Consulta privada & 36 & 28 & & & \\
\hline
\end{tabular}




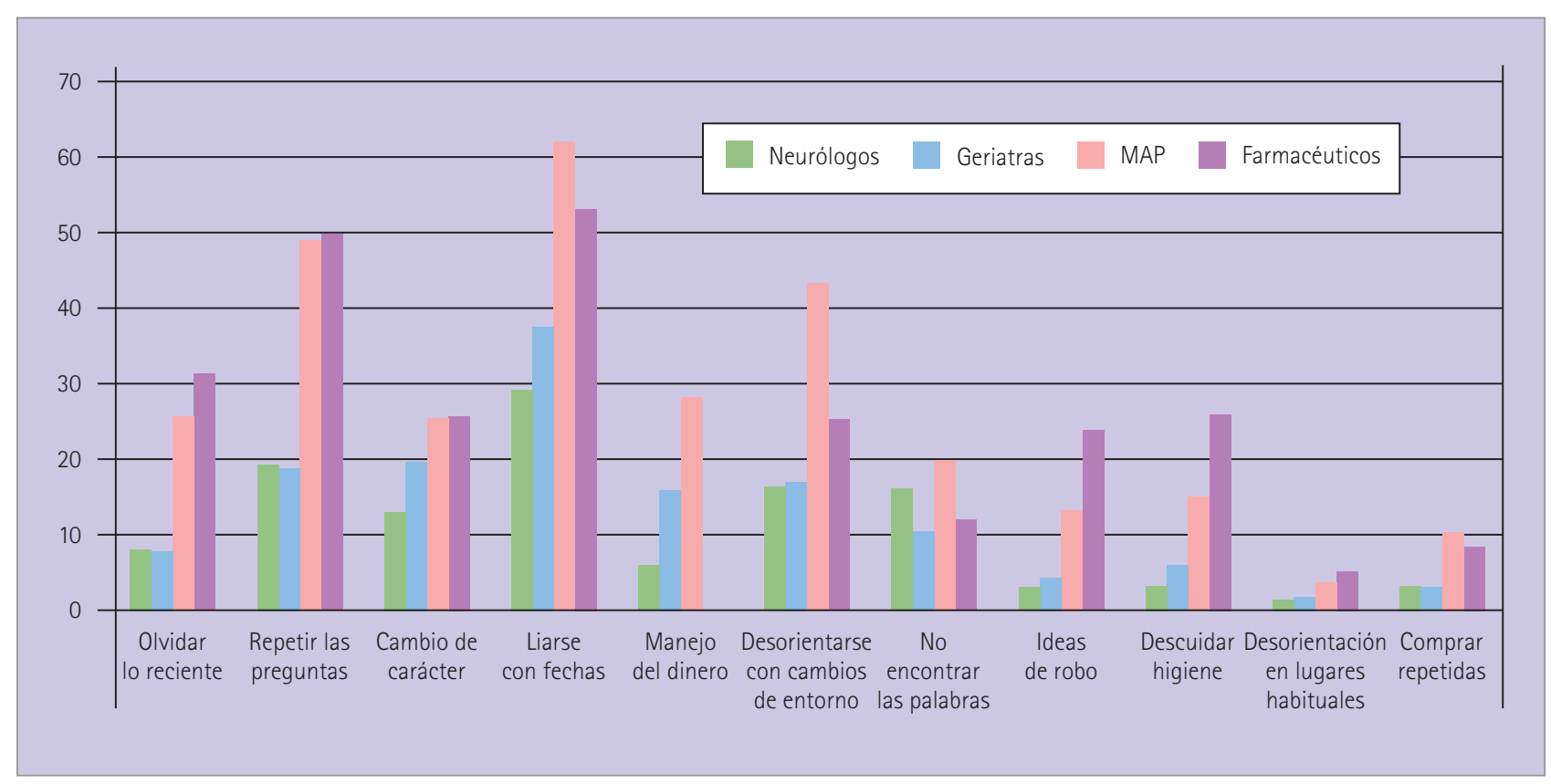

Figura 1 Porcentaje de profesionales que consideraron cada uno de los cambios iniciales descritos por los cuidadores como más propios del envejecimiento normal que del inicio de una $E A$

consulta de un paciente con deterioro cognitivo, un $22 \%$ de 20 minutos y un $20 \%$ de 15 minutos. Globalmente, entre un 20\% y un 30\% de los pacientes que acuden a la consulta por deterioro cognitivo tienen ya una demencia establecida cuando llegan a la consulta y el 51\% de los especialistas refieren que el tiempo medio de evolución de los síntomas es superior a un año en la primera consulta. Un 5\% de los neurólogos refiere que el tipo de demencia más frecuente en sus consultas es la demencia senil. Un porcentaje de especialistas consideraron propios del envejecimiento, y no posibles manifestaciones de una EA, sintomas como "repetirse en las mismas preguntas" (19\%), "desorientarse con un cambio de entorno" (16\%), "confundirse con las fechas" (30\%) o "no encontrar las palabras comunes" (16\%) (figura 1).

Las pruebas de cribado de deterioro cognitivo más utilizadas son el mini examen del estado mental (MMSE) (96\%), seguido del test del dibujo del reloj (84\%) y fluencia verbal (52\%). Un 68\% de los especialistas utiliza tests específicos para la memoria y otras funciones cognitivas, y un 61\% tiene acceso a solicitar una evaluación neuropsicológica. Un 95\% de los especialistas utiliza el concepto de "deterioro cognitivo ligero" como diagnóstico. Un 31\% manifestaba utilizar el diagnóstico de EA prodrómica o pre-demencia, aunque un 44\% afirmaba que lo utilizaría si tuviera la posibilidad de utilizar biomarcadores. Un 14\% estaba totalmente de acuerdo y un 53\% parcialmente de acuerdo en que los nuevos criterios NIA-AA (National Institute on Aging and the Alzheimer's Association) (18), que permiten hacer un diagnóstico de EA en fase de deterioro cognitivo ligero y EA preclínica, harán cambiar su actitud diagnóstica. Un 15\% opinaba que hacer un diagnóstico diferencial entre las distintas formas de demencia no es abordable (por la falta de acceso a pruebas neuropsicológicas o de neuroimagen) o carece de implicaciones prácticas.

Un 89\% de los neurólogos opinaba que los pacientes diagnosticados de deterioro cognitivo ligero son susceptibles de tratamiento con inhibidores de la acetilcolinesterasa si la sospecha de EA es alta. Respecto a la eficacia de los fármacos anti-Alzheimer, un 62\% manifestaba que mejoran síntomas cognitivos, un 57\% que estabilizan transitoriamente los síntomas de la enfermedad y un 52\% que mejoran la calidad de vida de paciente y cuidador (figura 2). El 94\% prescribe la terapia combinada con inhibidores de la acetilcolinesterasa y memantina en algún momento de la evolución.

Un 83\% de los neurólogos aseguró abordar aspectos sobre el pronóstico, síntomas conductuales, aspectos legales, aspectos del cuidador y otros cuando se comunica el diagnóstico de EA y un 13\% adicional afirmó que lo haría si tuviera tiempo en la consulta. Aproximadamente la mitad de los especialistas en Neurología reconocieron carecer de formación suficiente para valorar el estado nutricional y hacer recomendaciones de tipo nutricional o dietético. Los neurólogos reconocieron tener escasa información para poder asesorar sobre recursos sociosanitarios (34\%) o sobre temas de protección legal como incapacidad, poderes, etc. (50\%). En relación con la investigación, un 53\% de los neurólogos manifestaba su interés en participar en ensayos clínicos con nuevos fármacos para la EA y más de la mitad afirmaba que solicitaría a sus pacientes la donación de cerebros para investigación si supiera cómo hacerlo. La formación más demandada fue la que hacía referencia a los aspectos legales (18\%), bioética (16\%) y demencias no Alzheimer (15\%).

\section{Geriatras}

Los datos demográficos y el ámbito de actividad de los especialistas en Geriatría que respondieron a la encuesta se detallan en la tabla 1. Un $41 \%$ ejercía su actividad en unidades de memoria/demencias. Un 54\% de los encuestados reconocía que en más del 50\% de sus pacientes el motivo de consulta es deterioro cognitivo y un 35\% que más del 50\% de sus pacien- 


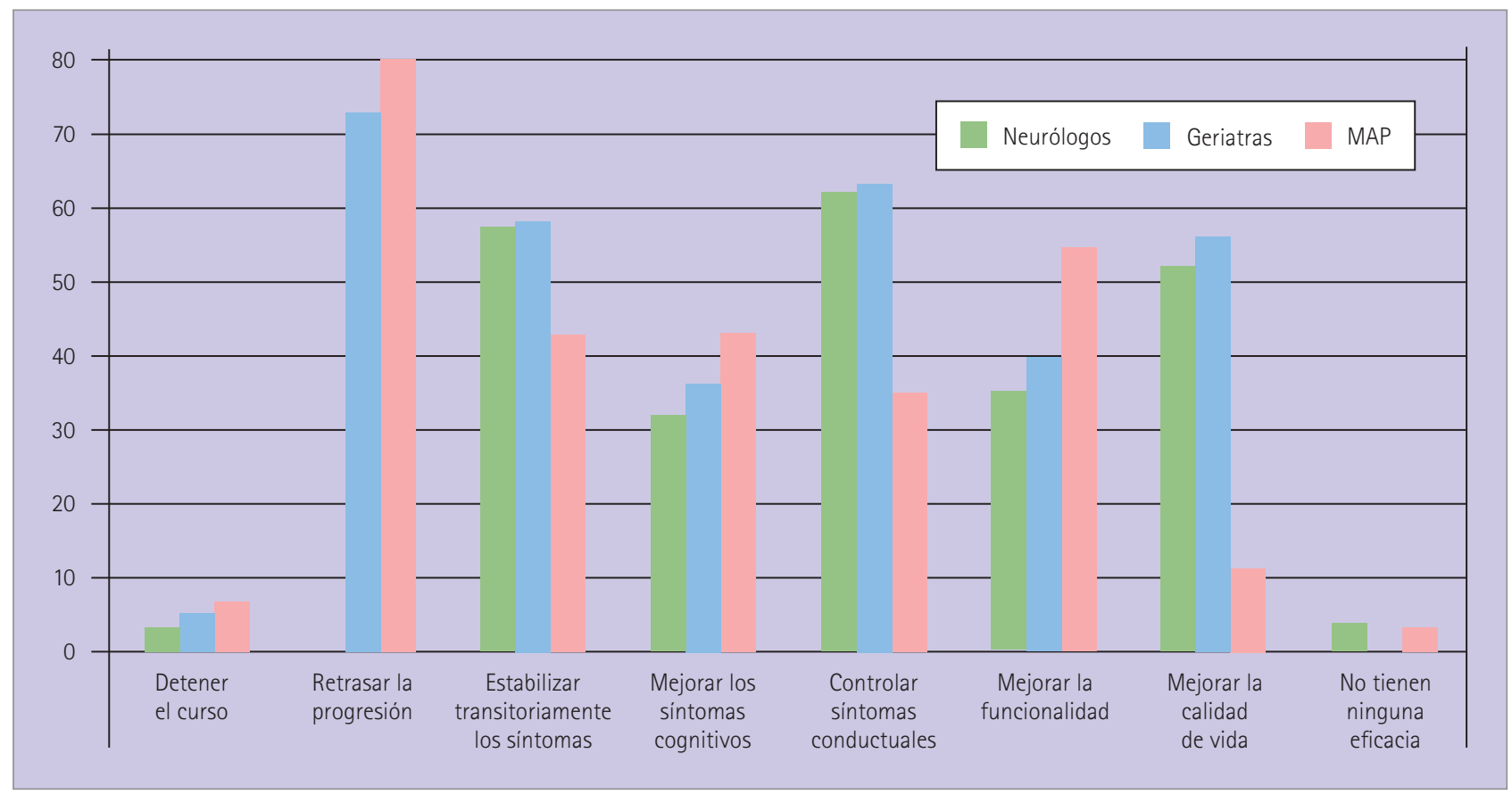

Figura 2 Percepción de la eficacia del tratamiento farmacológico de la EA en los grupos de profesionales (\%)

tes ya tiene un cuadro de demencia en la primera consulta. El tiempo medio de evolución de los síntomas referido era de 1 a 2 años (49\%) o superior a 2 años (15\%). Un 59\% de los geriatras dispone de 45 minutos o más para una primera consulta. Un $9 \%$ de los geriatras refiere que el tipo de demencia más frecuente en sus consultas es la "demencia senil". Un porcentaje de estos especialistas consideraron propios del envejecimiento y no posibles manifestaciones de una EA síntomas como "repetirse en las mismas preguntas" (19\%), "desorientarse con un cambio de entorno" (17\%), "confundirse con las fechas" (38\%) o "no encontrar las palabras comunes" (11\%) (figura 1). Las pruebas de cribado de deterioro cognitivo más utilizadas son el MMSE (92\%), seguida del test del dibujo del reloj (81\%), fluencia verbal (48\%) y test del informador (43\%). Un $71 \%$ de los especialistas en Geriatría utilizan tests específicos de memoria $\mathrm{u}$ otras funciones cognitivas.

El 92\% de los geriatras utiliza el concepto de deterioro cognitivo ligero entre sus diagnósticos y un 89\% opinó que los pacientes diagnosticados de deterioro cognitivo ligero son susceptibles de tratamiento con inhibidores de la acetilcolinesterasa si la sospecha de EA es alta. Un $20 \%$ estaba totalmente de acuerdo y un $42 \%$ parcialmente de acuerdo en que los nuevos criterios NIA-AA (17) harán cambiar su actitud diagnósti- ca. El 29\% aplica el diagnóstico de enfermedad de Alzheimer prodrómica y un 50\% lo utilizaría si tuviera acceso al uso de biomarcadores. Un 20\% de los encuestados opinaba que hacer un diagnóstico diferencial entre las distintas formas de demencia no es abordable por falta de acceso a pruebas neuropsicológicas o de neuroimagen, o no tiene implicaciones prácticas.

En la comunicación del diagnóstico, el 89\% aborda otros aspectos, además del mero diagnóstico. Un 4\% lo haría si tuviera tiempo de consulta. Respecto al tratamiento, un $84 \%$ estaba parcial (27\%) o totalmente (58\%) de acuerdo en prescribir inhibidores de la acetilcolinesterasa cuanto antes. La mayoría de los geriatras consideraba que los fármacos anti-Alzheimer retrasan el curso de la enfermedad (71\%), estabilizan transitoriamente los síntomas (58\%), controlan los síntomas conductuales (63\%) y mejoran la calidad de vida de paciente y cuidador (56\%) (figura 2). Un 88\% aplican la terapia combinada con inhibidores de la acetilcolinesterasa y memantina en algún momento de la evolución. Un 69\% afirmaba disponer de suficiente información para asesorar sobre el ingreso del paciente en un centro de día o centro gerontológico de larga estancia. Un 30\% opinaba que necesitaba más formación para asesorar en medidas de protección legal y un 51\% que precisaría saber más sobre cómo abordar dilemas éticos relacionados con el consentimiento informado. Un 72\% desearía participar en ensayos clínicos de nuevos fármacos. Asimismo, los encuestados manifestaban necesitar formación en aspectos legales (19\%), bioética (18\%) y demencias no Alzheimer (16\%).

\section{Médicos de atención primaria}

Los datos demográficos y el ámbito de actividad de los médicos de Atención Primaria (MAP) se detallan en la tabla 1. Entre los médicos de atención primaria (MAP) había un $59 \%$ de varones y el 40\% se encontraba en la franja de edad entre $50 \mathrm{y}$ 60 años. El 64\% de los encuestados afirmaba que los pacientes mayores de 65 años representan un 40\% o más de los pacientes que atienden. Un 54\% de los MAP encuestados refirieron que más del 20\% de las personas mayores de 65 años a las que ven viven solas. Un 25\% de los MAP opina que la demencia más frecuente entre sus pacientes es la demencia senil y un $27 \%$ está total o parcialmente de acuerdo en que la demencia senil es una forma más benigna de demencia que la EA. Tres de cada cuatro MAP disponen de menos de 10 minutos para valorar a un paciente con sospecha de deterioro cognitivo.

El 53\% de los participantes no había realizado ninguna actividad de formación (cursos, jornadas, talleres) 
sobre deterioro cognitivo en el último año. En los aspectos relativos a las necesidades de formación, destacaban el tratamiento, sintomas conductuales y psicológicos, así como demencias no EA. Algunos MAP reconocieron como más relacionados con el envejecimiento que con una EA sintomas como repetirse en las mismas preguntas (49\%), dificultad para manejar el dinero (28\%), desorientarse con un cambio de entorno (43\%), confundirse con las fechas (62\%), no encontrar las palabras comunes $(20 \%)$ o tener ideas delirantes de robo (13\%) (figura 1).

El $86 \%$ estaba parcial o totalmente de acuerdo en la necesidad de practicar una prueba de neuroimagen a todo paciente con deterioro cognitivo o demencia sin causa aparente, aunque el $46 \%$ no puede solicitarla. Un 24\% de los MAP cifró el tiempo de demora entre la solicitud de derivación y la cita con el especialista en 2 a 3 meses y un 24\% adicional estimó que era superior a 3 meses. Un 80\% de los MAP encuestados opina que los fármacos anti-Alzheimer retrasan la progresión de la enfermedad, controlan los sintomas conductuales (43\%) y mejoran la calidad de vida de paciente y cuidador (54\%) (figura 2). Un 58\% está parcial o totalmente de acuerdo en que los fármacos anti-Alzheimer se han de suspender a los 6 meses si no se observa una mejoría franca. No obstante, la mayoría está de acuerdo en que cuando un paciente no tolera o no responde a un determinado inhibidor de colinesterasa puede tolerar o responder con otro. El 54\% estaba de acuerdo en que los fármacos específicos anti-Alzheimer se deben retirar en fases avanzadas de demencia. En cuanto a los trastornos de conducta disruptivos, un 15\% de los MAP refiere consultar al psiquiatra, un 30\% al neurólogo y un 55\% instaura tratamiento sintomático. Entre estos últimos, prácticamente la mitad utilizan haloperidol u otro neuroléptico típico para tratar la agitación.

Aunque el 76\% de los encuestados estaban parcial (34\%) o totalmente $(42 \%)$ de acuerdo en que sería deseable derivar al especialista a todos los pacientes con deterioro cognitivo en los que no hay una causa tratable clara, en el 77\% de los casos no existe una vía rápida para la derivación y un 45\% no tiene acceso fácil (correo electrónico, teléfono) al especialista en caso de contingencias. El 64\% de los MAP declara que no existe una unidad de demencias en su área. Más de la mitad de los MAP visitan periódicamente en su domicilio a los pacientes con demencias avanzadas.

\section{Farmacéuticos}

Los datos demográficos de los farmacéuticos que respondieron a la encuesta se describen en la tabla 1. El 80\% de los encuestados refiere que más de un 50\% de la población que atiende es mayor de 60 años. El 51\% de los encuestados reconocía no tener ninguna formación sobre la EA. Solo un 9\% había asistido a un curso de formación de más de 20 horas. Un 75\% de los farmacéuticos opinaba que la demencia senil es la causa más frecuente de demencia en las personas mayores y el 47\% opinaba que ésta es una forma benigna de demencia.

Un porcentaje de los encuestados consideran propios del envejecimiento normal síntomas como tener fallos de memoria repetidos sobre los hechos recientes (23\%), necesitar ayuda en tareas cotidianas (31\%), repetirse en las cosas que se cuentan o se preguntan (50\%), desorientarse con un cambio de entorno (25\%) o tener ideas delirantes de robo (24\%) (figura 1).

Un 52\% de los farmacéuticos estaba de acuerdo en la afirmación de que los trastornos conductuales de la EA tienen tratamiento específico con psicofármacos y un 45\% opinaba que el tratamiento de elección en la agitación es el haloperidol. Uno de cada cuatro farmacéuticos opinaba que no existe tratamiento para la EA. Un $41 \%$ estaban parcial o totalmente de acuerdo en que el tratamiento con inhibidores de la acetilcolinesterasa ha de suspenderse cuando aparecen efectos adversos y un 27\% suspendería el tratamiento si no hay mejoría a los 6 meses. Un 89\% de los encuestados estaba total (67\%) o parcialmente (22\%) de acuerdo en que los tratamientos farmacológicos mejoran la calidad de vida de los pacientes (figura 2) y cuidadores y un 84\% está totalmente de acuerdo en que las terapias no farmacológicas (estimulación cognitiva, entrenamiento de memoria, etc.) son beneficiosas en la prevención y tratamiento de la EA. La mayoría de los farmacéuticos ofrece recomendaciones ante problemas relacionados con el cuidado del paciente con demencia como la desnutrición, incontinencia o úlceras de presión.

Un 40\% de los farmacéuticos conoce las herramientas de cribado para el diagnóstico de EA y cree que en coordinación con el médico podría aplicarlas en la farmacia. Igualmente, un porcentaje de los encuestados reconocían su desconocimiento en materias como el maltrato (71\%), incapacitación legal (57\%), herramientas para evaluar la carga del cuidador (48\%) o recursos sociosanitarios en su localidad (68\%). Respecto a los temas de formación más demandados destacaba la necesidad de disponer de protocolos actualizados y manejables sobre los tratamientos farmacológicos, guías nutricionales, así como información sobre detección precoz de EA, recursos para derivar a los posibles pacientes y orientar a los familiares, así como documentación consensuada por sociedades para el propio enfermo y el cuidador.

\section{Cuidadores/familiares}

La tabla 1 resume los datos demográficos del grupo de cuidadores que participaron en el proyecto. El $73 \%$ vive con la persona afectada de demencia y el 69\% como cuidador principal. Un $22 \%$ son esposas y un 59\% hijas de las personas enfermas a quienes cuidan y un 33\% de los encuestados llevaba más de 5 años cuidando al paciente. Un 17\% cuidaba de una persona con demencia en fase inicial, un 43\% en fase moderada y un 32\% en fase avanzada. El tipo de demencia era EA en un 77\% de los casos. Los síntomas iniciales que los cuidadores percibieron en sus familiares fueron fundamentalmente los fallos de memoria (80\%), olvidar lo reciente (50\%) o repetirse en las preguntas (48\%) y también otros no relacionados directamente con la memoria como los cambios de carácter (37\%), confundir fechas (35\%), liarse con el dinero (31\%) hacer "cosas raras" (30\%) o desorientarse con un cambio de entorno (29\%) (figura 3). En más de la mitad de los casos, el tiempo transcurrido entre la detección de esos primeros síntomas y la decisión de ir al médico fue superior a 6 meses. Entre los que tardaron más de 12 meses (20\%) la causa más frecuente fue que achacaron los sintomas a la edad (26\%) o a una depresión (22\%), 


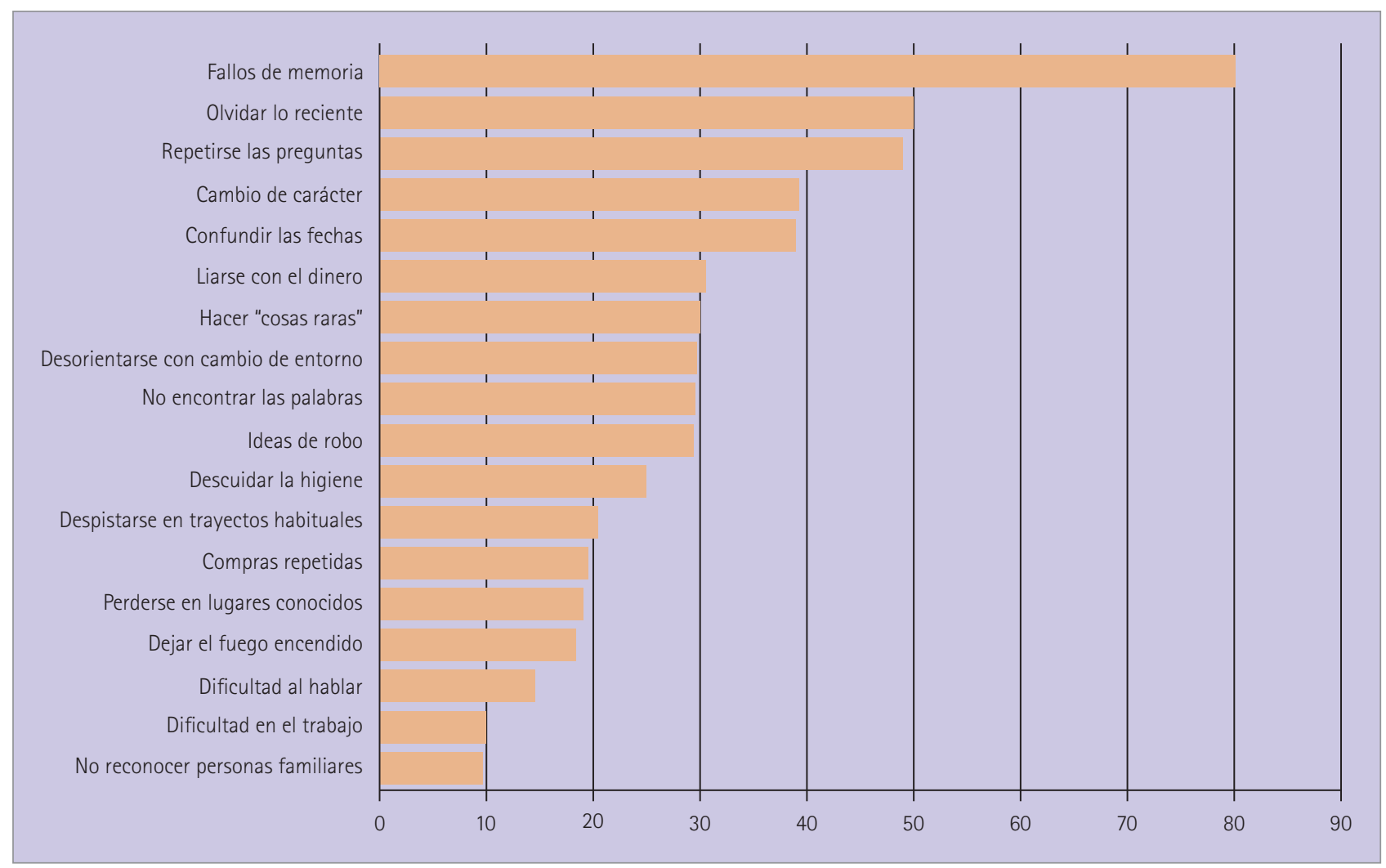

Figura 3 Cambios percibidos por los cuidadores como sintomas iniciales de la enfermedad (\% de cuidadores que observaron cada síntoma al inicio de la enfermedad en la persona a la que cuidan)

pero en un $18 \%$ de los casos fue un médico el que restó importancia a los sintomas y desaconsejó la consulta. El tiempo transcurrido entre la primera consulta y el diagnóstico fue superior a 6 meses en un 20\% de los casos y superior a 1 año en otro $20 \%$. El motivo del retraso más frecuente fueron las listas de espera (37\%) pero también otros como que el médico de cabecera no vio inicialmente motivo para derivar (17\%) o que se hicieron otros diagnósticos como demencia senil (13\%), depresión (13\%), nada (6\%) o que el especialista dijo que no estaba seguro y había que esperar y ver la evolución (8\%).

Un 31\% de los cuidadores sintió "alivio" al conocer el diagnóstico y un 33\% pudo "afrontarlo bien" en seguida, aunque se llevaran un "buen disgusto". Los cuidadores encuestados refirieron haber recibido información escasa o nula sobre aspectos como aspectos generales de la enfermedad y pronóstico (65\%), posibilidad de tratamiento no farmacológico (62\%), síntomas conductuales $(76 \%)$, recursos sociosanitarios (77\%), necesidades de cuidado del cuidador (81\%) o aspectos legales (86\%), Un 97\% y un 94\% de los cuidadores querrian saber cuanto antes si un familiar o ellos mismos tienen EA, respectivamente.

Tras el inicio del tratamiento con fármacos los cuidadores percibieron que la enfermedad mejoró un poco (17\%), mejoró mucho (5\%) o se estabilizó (44\%) (figura 4). Un 44\% de los cuidadores cree que los síntomas conductuales de la EA no tienen un tratamiento específico. Un $47 \%$ de los cuidadores opinaban que su salud había empeorado por tener que cuidar a su familiar y un 54\% afirmó que la enfermedad había tenido efectos negativos en el seno familiar. La administración de cuidados básicos, los síntomas conductuales y la necesidad de restringir actividades constituyeron las principales dificultades y fuentes de sobrecarga. Un 55\% de cuidadores carecía de una idea clara de las voluntades de la persona enferma para la toma de decisiones al final de la vida y un $62 \%$ ignoraba la existencia de los documentos de instrucciones previas o directrices anticipadas. Globalmente, un $60 \%$ de encuestados manifestó carecer de información acerca de numerosos aspectos de la EA y recursos disponibles (tabla 2) y un $86 \%$ desconocía la posibilidad de donación de tejidos neurológicos.

\section{Discusión}

Los resultados del proyecto $k N O W$ Alzheimer ofrecen un retrato actualizado de la realidad de la atención a los pacientes con EA en España en el contexto profesional y familiar y brindan una oportunidad inmejorable para poner en manos de estos colectivos, y de la sociedad en general, la información necesaria en aspectos en los que se detectan lagunas y necesidades de conocimiento.

A fin de poder ofrecer respuestas concretas, el objetivo del estudio era recopilar información sobre actitudes, dudas reales, controversias y puntos susceptibles de mejora relativos al diagnóstico, cuidado y tratamiento de la EA en los distintos colectivos implicados. La información derivada de este estudio es novedosa y de gran interés. Por primera vez se ha pulsado la opinión de neurólogos, geriatras, médicos de atención primaria, farmacéuticos y cuidadores/familiares como amplia muestra de los colectivos que en la vida diaria participan en la atención y el cuidado del paciente con EA.

Los datos obtenidos en este trabajo son difícilmente comparables con estudios previos. En España no se ha llevado a cabo un trabajo de características similares. En el ámbito de 
Tabla 2 Déficits de información y desconocimiento sobre diversos aspectos relacionados con la enfermedad de Alzheimer (AE) manifestados por el colectivo de cuidadores/familiares

\begin{tabular}{|l|c|c|}
\hline \multirow{2}{*}{$\begin{array}{l}\text { ¿Conoce en qué consiste...? o ¿tiene idea de que existe...? } \\
\text { ¿sabe qué es ...? }\end{array}$} & Si & No \\
\hline Manejo del cuidado del paciente con EA & 43 & 57 \\
\hline $\begin{array}{l}\text { Trastornos debidos a problemas de memoria/conducta/cambios } \\
\text { estados de ánimo }\end{array}$ & 28 & 49 \\
\hline Servicios de ayuda a domicilio de los servicios municipales & 45 & 55 \\
\hline Servicio de teleasistencia & 30 & 51 \\
\hline Hospital de día & 28 & 60 \\
\hline Cuidador interino & 20 & 60 \\
\hline Servicio de residencia & 17 & 49 \\
\hline Asociación de familiares & 56 & 28 \\
\hline Ingreso de respiro familiar & 11 & 70 \\
\hline $\begin{array}{l}\text { Ingreso en un centro sociosanitario (convalecencia o control de } \\
\text { sintomas) }\end{array}$ & 19 & 71 \\
\hline Soporte psicológico & 23 & 59 \\
\hline Talleres o programas de mantenimiento de memoria & 48 & 36 \\
\hline $\begin{array}{l}\text { Programas de vacaciones terapéuticas para afectados y sus } \\
\text { familiares }\end{array}$ & 7 & 75 \\
\hline Ayuda por parte de voluntarios & 11 & 71 \\
\hline $\begin{array}{l}\text { Documento de instrucciones previas, directrices anticipadas o } \\
\text { testamento vital }\end{array}$ & 38 & 62 \\
\hline $\begin{array}{l}\text { Donación de órganos y tejidos, en concreto banco de tejidos } \\
\text { neurológicos }\end{array}$ & 14 & 86 \\
\hline Ensayo clinico & 37 & 63 \\
\hline Datos expresas & & \\
\hline
\end{tabular}

Datos expresados en porcentajes (total: 858 cuidadores/familiares).

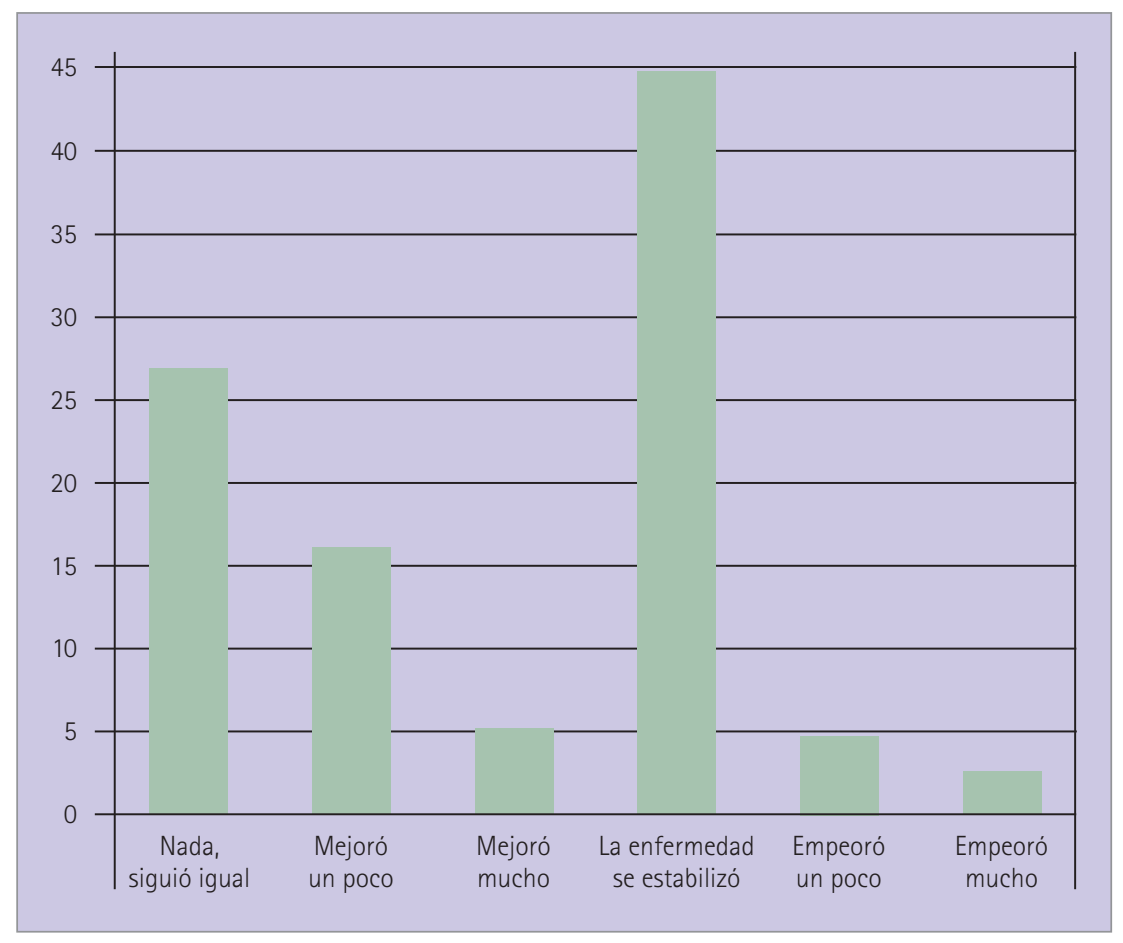

Figura 4 Percepción de la eficacia del tratamiento farmacológico de la EA según los cuidadores (\%) la Unión Europea, el estudio IMPACT (Important Perspectives on Alzheimer's Care and Treatment) (19) evaluó a través de una encuesta el grado información y las actitudes frente a la demencia en general, y la EA en particular, entre colectivos clave de cinco países: Francia, Alemania, Italia, España y Reino Unido. Participaron en la encuesta 1.700 personas, incluyendo 950 sujetos de la población general, 500 médicos, 250 cuidadores y 50 gestores de salud pública responsables de la toma de decisiones. Dicho estudio reveló que en opinión de los médicos hay un elevado nivel de infradiagnóstico en las fases tempranas, pese a que el 74\% de los médicos y el 63\% de los cuidadores consideran que un tratamiento a tiempo podría contribuir a retrasar los síntomas de la enfermedad (3). Asimismo, un elevado porcentaje de todos los grupos encuestados consideraba el cribado rutinario para el diagnóstico de EA importante o extremadamente importante (20). En opinión de los profesionales, parte del infradiagnóstico podría tener su origen en la incertidumbre de los médicos para diagnosticar en fases tempranas, la tendencia natural de pacientes y familiares a no valorar (o ignorar) los primeros sintomas, la actitud nihilista de los médicos que infravaloran un diagnóstico precoz dado que no existen tratamientos efectivos y las barreras emocionales asociadas a la tesitura de "dar malas noticias". El comité de expertos del estudio IMPACT recomendó el desarrollo de programas que ayudasen a los médicos a superar los desafíos asociados al diagnóstico y a mejorar el conocimiento entre los propios profesionales y la población general de los signos tempranos indicativos de la EA. En este sentido, el estudio kNOW Alzheimer ha puesto de manifiesto datos de interés. Un porcentaje no desdeñable de profesionales considera propios de la edad síntomas que buena parte de cuidadores han señalado como primeras manifestaciones de la enfermedad (figuras 1 y 3). Además, sigue utilizándose con excesiva frecuencia el diagnóstico de "demencia senil" al que además se da un carácter de benignidad. Los datos del estudio kNOW Alzheimer deben ayudar a los médicos a perder el miedo a "dar malas noticias". No solo la gran mayoría de los encuestados afirmaron querer saber cuanto 
antes si ellos mismos o alguien de la familia tienen EA, sino que el diagnóstico produce alivio y permite encarar el cuidado del enfermo. Por último, tampoco podrá esgrimirse como excusa del retraso en el diagnóstico el hecho de que la EA no tenga tratamiento. La gran mayoría de médicos especialistas y de atención primaria defienden la eficacia de los fármacos inhibidores de colinesterasa y memantina y el 75\% de los cuidadores encuestados percibieron mejoría o estabilización de los síntomas cuando estos fármacos fueron prescritos. Si el estudio kNOW Alzheimer contribuye a corregir las lagunas existentes en la formación y cambiar las actitudes puestas de manifiesto por usuarios y profesionales, podrán mejorarse sin duda datos como los aportados por el estudio de Elizegi y colaboradores, que mostró cómo de entre las personas con deterioro cognitivo evidente (21), sólo la mitad habían recibido un diagnóstico.

Entre los colectivos médicos ha de destacarse el escasísimo tiempo que se puede dedicar a una consulta por deterioro cognitivo. Se interpreta como positivo el amplio uso de la etiqueta diagnóstica de deterioro cognitivo ligero, pero se han de dar pasos para facilitar el diagnóstico en los momentos prodrómicos o de pre-demencia, facilitando el acceso al uso de biomarcadores (18). Esto puede ser especialmente importante si se pretende reducir el tiempo que transcurre entre el inicio de los síntomas y el momento en que los pacientes acuden a una primera consulta. El colectivo de cuidadores/familiares expresó que este tiempo fue superior a los 6 meses. Neurólogos y geriatras afirmaron que buena parte de sus pacientes acuden dentro de los primeros seis meses, aunque todavía se acercan al 50\% los que llevan más de un año de evolución. En un estudio multicéntrico, observacional y transversal, en el que se incluyeron 1.694 pacientes con EA probable atendidos en consultas de Atención Especializada en España, el tiempo medio ( \pm desviación estándar) desde los primeros síntomas a la primera consulta era de $10,9 \pm 17,2$ meses $\mathrm{y}$ hasta el diagnóstico de la EA de 28,4 $\pm 21,3$ meses (4). Estos datos, asimismo, concuerdan con la opinión del colectivo de geriatras, en el que el tiempo medio de evolución de los síntomas en la primera consulta era superior a uno o dos años. Se ha de trabajar también en la reducción de los tiempos de demora en la derivación, realización de pruebas diagnósticas y recepción del diagnóstico, tal como han expresado cuidadores $\mathrm{y}$ MAP en este sondeo.

Neurólogos, geriatras y médicos de atención primaria demostraron un buen conocimiento del tratamiento farmacológico, así como del uso combinado de inhibidores de la acetilcolinesterasa y memantina (94\% de los médicos). La percepción general entre los colectivos de profesionales y en los cuidadores es que los fármacos anti-Alzheimer aportan beneficio. Es de destacar el dato de que la gran mayoría de cuidadores (91\%) afirmaron que la persona a la que cuidaban había recibido algún tipo de tratamiento. Este hecho contrasta con otros referidos en la literatura, según los cuales menos de la mitad de los enfermos con demencia, aun estando diagnosticados, reciben tratamiento $(21,22)$. En una revisión sistemática de la literatura médica se confirman los beneficios clínicos de la memantina en las alteraciones de la capacidad para realizar actividades de la vida diaria en pacientes con EA (23). En este sentido, tras el análisis de los resultados se ha de insistir en la importancia de iniciar cuanto antes el tratamiento, cambiar la percepción sobre la eficacia de los fármacos anti-Alzheimer y ofrecer información con datos basados en la evidencia, poner a su disposición información ampliada sobre los inhibidores de la acetilcolinesterasa y otros fármacos en el control de los síntomas conductuales, pautas a seguir en caso de cambiar o suspender el tratamiento anti-Alzheimer, así como informar sobre el tratamiento en fases avanzadas. Asimismo, en el ámbito de la atención primaria parece necesario mejorar la formación acerca del manejo de los síntomas conductuales y psicológicos. El uso del haloperidol parece todavía demasiado extendido. Además, es imperativo mejorar los canales existentes de derivación del paciente e implantar una vía de contacto inmediato con la atención especializada en caso de surgir algunas contingencias.

La implicación del colectivo de farmacéuticos es un aspecto novedo- so de este estudio. Estos profesionales representan una parte importante del sistema de atención y cuidados a los pacientes con demencia, quizás aquella a la que los usuarios tienen un acceso más directo. El conocimiento de aspectos relacionados con detección de los primeros sintomas, tratamientos anti-Alzheimer y manejo de síntomas conductuales puede mejorar en este contexto. Demasiados opinan todavía que la demencia más frecuente es la demencia senil y uno de cada cuatro profesionales opinaba que la EA no tiene tratamiento. Resulta alentador, no obstante, el hecho de que un 40\% de ellos conocía las herramientas de cribado para el diagnóstico y estuvieran dispuestos a aplicarlos en personas con sintomas y en estrecha coordinación con los médicos. Al igual que en el resto de colectivos encuestados, se precisa también formación en cuestiones legales, aspectos relacionados con el cuidado y recursos sociosanitarios disponibles en su entorno.

En el ámbito del colectivo de cuidadores/familiares, un 60\% reconocía no haber recibido suficiente información. Este hecho contrasta con el expresado por neurólogos y geriatras que afirman que en el momento de comunicación del diagnóstico abordan todos los aspectos de la enfermedad (evolución, problemas de conducta, tratamiento, aspectos legales y recursos sociosanitarios, etc.). Parece importante, por tanto, diseñar estrategias que aporten a los cuidadores información y asesoramiento adecuados. Este proyecto kNOW Alzheimer ha confirmado una vez más que cuidar a una persona con EA tiene efectos negativos sobre la salud del cuidador y sobre la familia. El estudio ha resaltado también que las principales fuentes de sobrecarga son el desconocimiento, los síntomas conductuales y, como dato novedoso, la falta de información sobre estrategias de cómo afrontar los sintomas cognitivos y conductuales y cómo abordar la necesidad de restringir actividades en las que el paciente ya no es autónomo. Un diagnóstico más temprano, un tratamiento a tiempo y una información adecuada ayudarán sin duda a paliar estos efectos. En un estudio para evaluar el impacto en la calidad de vida relacionada con la salud, el tiempo de dedicación y la carga percibida 
por los cuidadores principales informales ( $n=268$ ) de los enfermos de EA en España, los cuidadores presentaron una peor calidad de vida en el cuestionario EuroQoL-5D que la población general. El 84\% de los cuidadores presentaba problemas de salud físicos y el 94,4\%, psicológico. Además, el 46,5\% tenía una carga entre moderada y severa, y el 34,7\%, una sobrecarga severa. Sólo el 26,9\% recibió alguna ayuda sociosanitaria y el 76,5\%, algún tipo de ayuda de las asociaciones de familiares de Alzheimer (24). Asimismo, las intervenciones educativas dirigidas a cuidadores de pacientes con EA, no tan solo han demostrado un aumento notable del conocimiento en el manejo de los pacientes, sino también un progreso significativo en la relación cuidador-enfermo (25).

Los resultados de este estudio deben interpretarse teniendo en cuenta las limitaciones del mismo, básicamente referidas al número de participantes que cumplimentaron todos los apartados de la encuesta. Por otra parte, también se desconoce el número exacto de miembros de cada colectivo con autorización para recibir información por correo electrónico, lo cual no permite una estimación del porcentaje de participantes en relación al universo de personas supuestamente contactadas. Si bien puede argumentarse que la muestra estudiada es relativamente reducida, por otra parte, podría reflejar la selección natural de las personas con mayor interés en el tema de la EA, en especial si se consideran las características de las preguntas y la extensión de los respectivos cuestionarios. Además, el hecho de que los cuestionarios fueran desarrollados y consensuados por un panel de expertos de cada sociedad y que el porcentaje de participantes sobre el total de personas de cada colectivo registradas en la plataforma fuera superior al 70\% (a excepción del 55\% en el caso de los geriatras), apoyan la validez de los resultados obtenidos.

En resumen, gracias a la puesta en práctica del proyecto kNOW Alzheimer ha sido posible obtener información actualizada acerca de las actitudes, dudas y conocimientos de los distintos colectivos involucrados en la atención de los enfermos con EA. Como resultado se han puesto en evidencia necesidades específicas de formación, lo que, sin duda, redundará en el diseño de estrategias dirigidas a subsanar dichas carencias.

\section{Referencias bibliográficas}

1. De Pedro-Cuesta J, Virués-Ortega J, Vega S, Seijo-Martínez M, Saz $\mathrm{P}$, Rodriguez F et al. Prevalence of dementia and major dementia subtypes in Spanish populations: a reanalysis of dementia prevalence surveys, 1990-2008. BMC Neurol. 2009; 9:55. doi:10.1186/1471-23779-55

2. Informe Estado del Arte de la Enfermedad del Alzheimer en España. Madrid: PwC; 2013.

3. Martinez-Lage P, Frölich L, Knox S, Berthet K. Assessing physician attitudes and perceptions of Alzheimer's disease across Europe. J Nutr Health Aging. 2010; 14:537-44. doi:10.1007/ s12603-010-0265-9

4. Alom Poveda J, M. Baquero JM, González-Adalid Guerreiro M. Estadio evolutivo de los pacientes con enfermedad de Alzheimer que acuden a la consulta especializada en España. Neurología. 2012. doi:10.1016/j. nrl.2012.10.003.

5. Blennow K, Zetterberg $\mathrm{H}$. The application of cerebrospinal fluid biomarkers in early diagnosis of Alzheimer disease. Med Clin North Am. 2013; 97:369-76. doi:10.1016/j. mcna.2012.12.012

6. Rowe CC, Villemagne VL. Amyloid imaging with PET in early Alzheimer disease diagnosis. Med Clin North Am. 2013; 97:377-98. doi:10.1016/j. mcna.2012.12.017

7. Salloway S, Correia S. Alzheimer disease: time to improve its diagnosis and treatment. Cleve Clin J Med. 2009; 76:49-58. doi:10.3949/ ccjm.76a.072178

8. Solomon PR, Murphy CA. Early diagnosis and treatment of Alzheimer's disease. Expert Rev Neurother. 2008; 8:769-80. doi:10.1586/ 14737175.8.5.769

9. Mori T, Maeda J, Shimada H, Higuchi M, Shinotoh H, Ueno S, et al. Molecular imaging of dementia. Psychogeriatrics. 2012; 12:106-14. doi:10.1111/ j.1479-8301.2012.00409.x

10. Criado-Álvarez JJ, Romo Barrientos C. Variabilidad y tendencias en el uso de fármacos contra la demencia. Neurología. 2010; 25:234-8. doi:10.1016/j. nrl.2009.12.003

11. Villar Fernández I, Rabanaque Hernández MJ, Armesto Gómez J, García Arilla E, Izuel Rami M. Utilización de fármacos específicos para la enfermedad de Alzheimer. Neurología. 2007; 22:275-84.
12. Gil-Néciga E, Gobartt AL. Patrón de tratamiento de la enfermedad de Alzheimer con inhibidores colinesterásicos (estudio TRAIN). Rev Neurol. 2008; 46:461-4.

13. Leuzy A, Gauthier S. Ethical issues in Alzheimer's disease: an overview. Expert Rev Neurother. 2012; 12:557-67. doi:10.1586/ern.12.38

14. Villars H, Oustric S, Andrieu S, Baeyens JP, Bernabei R, Brodaty $\mathrm{H}$, et al. The primary care physician and Alzheimer's disease: an international position paper. J Nutr Health Aging. 2010; 14:110-20. doi:10.1007/s12603010-0022-0

15. Nourhashémi F, Gillette $S$, Cantet C, Stilmunkes A, Saffon N, Rougé-Bugat $\mathrm{ME}$, et al. End-of-life care for persons with advanced Alzheimer disease: design and baseline data from the ALFINE study. J Nutr Health Aging. 2012; 16:457-61. doi:10.1007/ s12603-011-0333-9

16. European Parliament resolution of 19 January 2011 on a European initiative on Alzheimer's disease and other dementias (2010/2084(INI).

17. Council of the European Union. Council conclusions on public health strategies to combat neurodegenerative diseases associated with ageing and in particular Alzheimer's disease. $2916^{\text {th }}$ Employment, Social Policy, Health and Consumer Affairs Council Meeting. Brussels, 16 December 2008.

18. McKhann GM, Knopman DS, Chertkow H, Hyman BT, Jack CR, Kawas, $\mathrm{CH}$, et al. The diagnosis of dementia due to Alzheimer's disease: recommendations from the National Institute on Aging and the Alzheimer's Association workgroup. Alzheimer's Dement. 2011; 7:263-9. doi:10.1016/j. jalz.2011.03.005

19. Jones RW, Mackell J, Berthet K, Knox S. Assessing attitudes and behaviours surrounding Alzheimer's disease in Europe: key findings of the important perspectives on Alzheimer's care and treatment (IMPACT) Survey. Nutr Health Aging. 2010; 14:525-30.

20. Bond J, Graham N, Padovani A, Mackell J, Knox S, Atkinson J. Screening for cognitive impairment, Alzheimer's disease and other dementias: opinions of European caregivers, payors, physicians and the general public. J Nutr Health Aging. 2010; 14:558-62. doi:10.1007/s12603-0100268-6

21. Elizegi-Igerategi M, Arriola-Manchola E, Sánchez M. Análisis de la demanda de recursos sociales en relación al deterioro cognitivo. Psicogeriatría 2010; 2:25-33.

22. Grupo de Estudio de la Demencias de la Sociedad Española de Geriatría y 
Gerontología. Estudio DECADA-G: Demencia en España, Calidad asistencial y demanda atendida por la Geriatría. Informe Final. Madrid: Sociedad Española de Geriatría y Gerontología; 2005.

23. López Arrieta JM. Beneficios clínicos de la memantina en las alteraciones de la capacidad para realizar actividades de la vida diaria en pacientes con enfermedad de Alzheimer. Revisión sistemática de la bibliografía médica. Rev Esp Geriatr Gerontol. 2006; 41:1839. doi:10.1016/S0211-139X(06)729 50-X

24. Badia Llach X, Lara Suriñach N Roset Gamisans, M. Calidad de vida, tiempo de dedicación y carga percibida por el cuidador principal informal del en- fermo de Alzheimer. Aten Primaria. 2004; 34:170-7. doi:10.1016/S02126567(04)78904-0

25. Alcaraz Agüero M, Labori Ruiz JR, Font A, Alayo Blanco M, García IR. Modificación de conocimientos en los cuidadores de pacientes con enfermedad de Alzheimer. Aten Primaria. 2009; 41:350. doi:10.1016/j. aprim.2008.10.012 